\title{
Pseudorandom numbers for mini- and microcomputers: A generalized feedback shift register algorithm
}

\author{
W. H. PAYNE* \\ Department of Computer Science \\ ILLIAC III Project \\ University of Illinois at Urbana-Champaign \\ Urbana, Illinois 61801
}

The advantages of the generalized feedback shift register pseudorandom number algorithm over all other pseudorandom number generators are described. These advantages are: (1) It produces multidimensional pseudorandom numbers; (2) it has arbitrarily long period, independent of word size of the computer on which it is implemented; (3) it is faster than other pseudorandom number generators; (4) the "same" floating-point pseudorandom number sequence is obtained on any machine; that is, the high-order mantissa bits of each pseudorandom number agree on all machines: examples are given for IBM 360 , SPERRY-RAND-UNIVAC 1108 , CONTROL DATA 6000 , and HEWLETT-PACKARD 2100 series computers; and (5) it can be coded in compiler languages (is portable). This pseudorandom number generator is ideal for mini- and microcomputers, since only two additions and an exclusive-or are needed for its execution.

The Lehmer congruential multiplicative pseudorandom number generator has been shown to have n-space nonuniformity (Conveyou \& Macpherson, p-bit words are decimated into n subwords (Tausworthe, 1965 ). Figures 1 and 2 give examples of nonuniformity of Lehmer RNGs for 9-bit words in two- and three-space. FSR generators with primitive generating polynomial, $X^{p}+X^{q}+1$, small $q$ or $q$ near $(p-1) / 2$, should be avoided because of bad runs properties (Tootill, Robinson, \& Adams, 1971). However, careful selection of $p, q$ provides satisfactory random numbers in low-dimensional space (Whittlesey, 1968).

Perhaps FSR sequences offer the best prospects for n-space improvement. Kendall's algorithm is moderately fast on most machines, but the period is fixed by the word size and it is difficult to implement in multiprecision (Whittlesey, 1968). Moreover, decimation in order to gain $n$-space uniformity further shortens cycle length and resolution. This problem is intrinsic to periodic sequences. A cyclic sequence of $m$ numbers, when taken in pairs, locates only $\mathrm{m}$ of $\mathrm{m}^{2}$ points in a two-dimensional $\mathrm{m}$ by $\mathrm{m}$ grid. In general, $\mathrm{m}^{\mathfrak{n}}$-m grid points will never be located in $n$ space by n-tuples taken from an m-periodic sequence, i.e., most cross products are missing. This sparseness in $n$ space underlies nonuniformity of all periodic RNGs. Apparently, what is needed is a RNG that allows repeated numbers within a full period sequence. Such repeated numbers could fill in

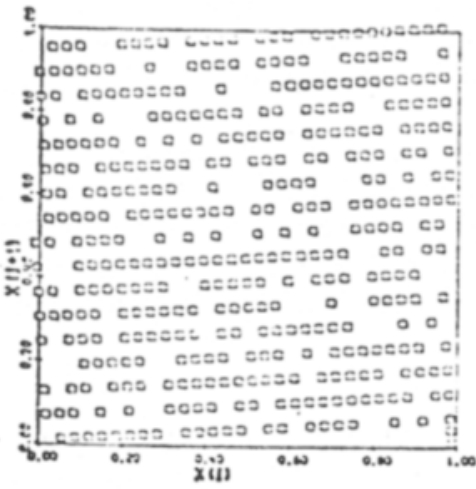

(a)

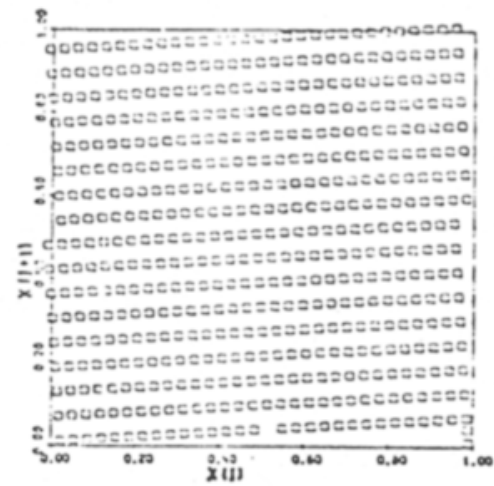

(b)

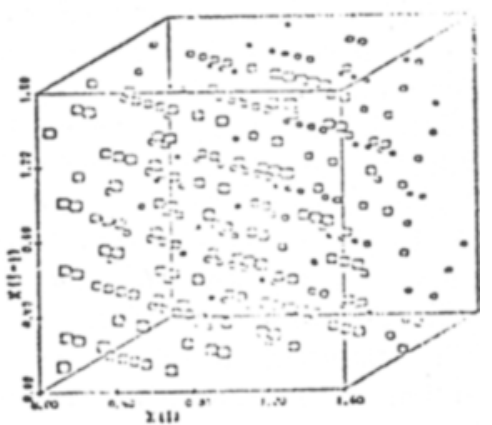

(c)

Fig. 1. Continued growth of "crystals" in $X_{i+1}=17 X_{i}-1$ (Mod 512): (a) 384 points, (b) 512 points in 2-space, and (c) plot in 3-space of 256 successive triplets.

1967; Marsaglia, 1968). This shortcoming is particularly severe for small-word machines. As an alternative, the feedback shift register (FSR) pseudorandon number generator (RNG) is claimed to be uniform in $n$ space if

\footnotetext{
*Visiting Research Associate Professor of Computer Science on leave from the Department of Computer Science, Washington State University, Pullman, Washington 99163
}

$m^{n}$ points, for some $n$.

The purpose of this paper is to make the attendants of this conference familiar with a new, completely general FSR algorithm for generating arbitrarily long sequences of random numbers possessing desirable n-space properties on any word-size machine (Lewis \& Payne. 1973). The algorithm is idcally suited for generation of 


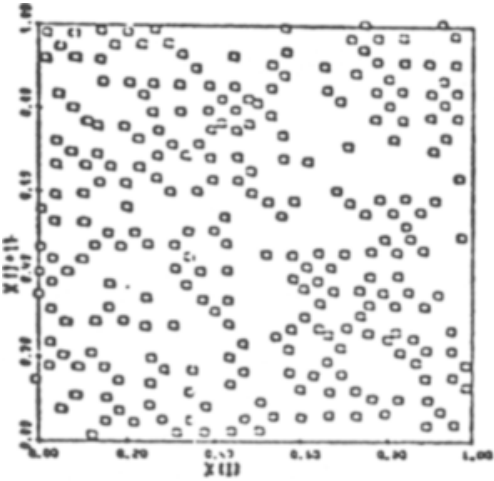

(a)

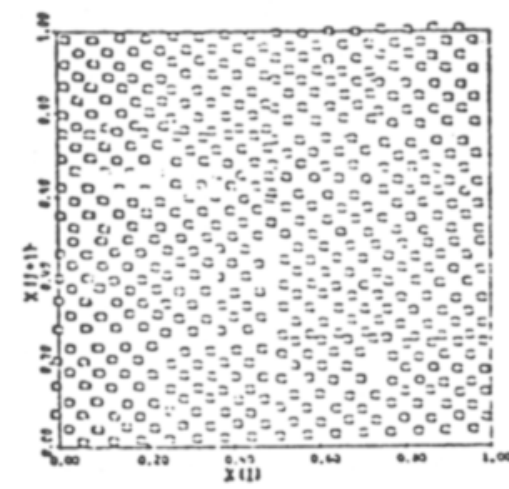

(c)

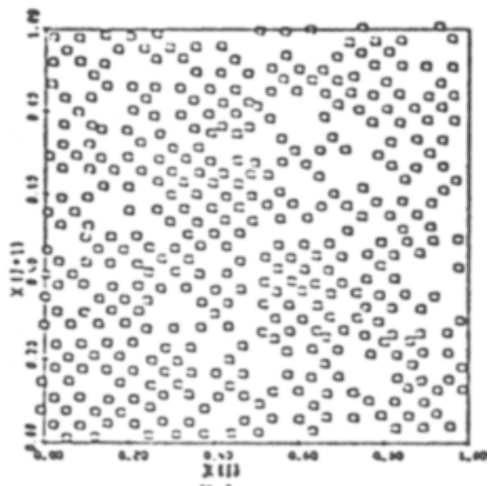

(b)

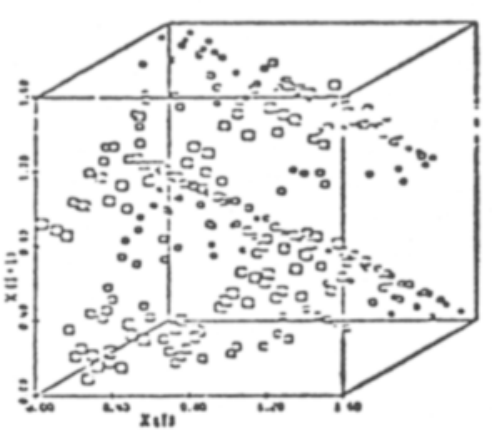

(d)
Fig. 2. RNG FSR: $X^{9}+X^{4}+1$. (a) 127 , (b) 255 , (c) 511 points plotted (successive pairs) in 2-space, and (d) 255 successive 3-tuples plotted in 3-space. pseudorandom numbers on mini- and microcomputers in real time.

\section{GENERALIZED FEEDBACK SHIFT REGISTER ALGORITHM (GFSR)}

Define $\oplus$ to be the "exclusive-or" operator which is equivalent to addition modulo 2 . Kendall's algorithm is used to select successive n-tuples from the basic sequence $\left(a_{i}\right)$, where $a_{k}=a_{k-p+q} \oplus a_{k-p}, k=p, p+1$, $\cdots$, given $a_{p-1}, \cdots, a_{0}$ and feedback shift register based on primitive polynomial $x^{p}+x^{q}+1(10)$. For example, $\mathrm{x}^{5}+\mathrm{x}^{2}+1$ and $\mathrm{a}_{0}=\mathrm{a}_{1}=\mathrm{a}_{2}=\mathrm{a}_{3}=\mathrm{a}_{4}=1$ yields $\left(\mathrm{a}_{\mathrm{i}}\right)_{0}^{30}$

$=1111100011011101010000100101100$. Selecting 5-tuples, $A_{i} s$ by Kendall's algorithm produces the random numbers seen in Fig. 3.

It is important to observe that each $A_{i}$ (with the exception of 0 ) occurs once and only once in the full period of a Tausworthe pseudorandom sequence (the "Kendall sequence" is one specific "Tausworthe sequence").

The idea behind the genralized feedback shift register pseudorandom number algorithm (GFSR) is that the basic shift register sequence $\left(a_{1}\right)$ based on primitive trinomial $x^{p}+x^{q}+1$ is set into $j$ columns, $j<p$, with a judiciously selected delay between columns. An example will make the basic GFSR algorithm clear. Again, choose primitive trinomial $x^{5}+x^{2}+1$. The basic sequence of $\left(a_{i}\right)$ is copies in the first column of Fig. 4. For this particular polynomial, the second column was formed by delaying the first column by $25(25-31=-6$, depending on the orientation of the "circle" of bits) bit positions; the third column was obtained by delaying the second column by another -6 bit positions; and so on until all five columns have been filled.

Since each column obeys the recurrence $a_{k}=a_{k-p+q}$ $\oplus a_{k-p}$, each word must also obey $W_{k}=W_{k-p+q} \oplus$ $W_{k-p}$.

Carefully observe that each $W_{i}$ occurred once and only once in the full period of $2^{5}-1=31$ numbers.

Do arbitrary delays between the columns ensure that each number between 1 and $2^{p}-1$ occurs once and only once in each period? No, but any "starting matrix"

$\begin{array}{lllllllllllllllllll}A_{0} & 1 & 1 & 1 & 1 & 1 & A_{10} & 0 & 1 & 0 & 0 & 0 & A_{20} & 1 & 0 & 1 & 1 & 0 \\ A_{1} & 1 & 1 & 0 & 0 & 0 & A_{11} & 1 & 1 & 0 & 1 & 0 & A_{21} & 0 & 1 & 0 & 1 & 1 \\ A_{2} & 0 & 1 & 1 & 1 & 0 & A_{12} & 1 & 1 & 1 & 0 & 0 & A_{22} & 0 & 0 & 0 & 0 & 1 \\ A_{3} & 0 & 0 & 1 & 0 & 1 & A_{13} & 0 & 0 & 0 & 1 & 1 & A_{23} & 0 & 1 & 0 & 0 & 1 \\ A_{4} & 0 & 0 & 1 & 0 & 0 & A_{14} & 1 & 1 & 0 & 1 & 1 & A_{24} & 1 & 0 & 0 & 1 & 1 \\ A_{5} & 0 & 1 & 1 & 0 & 1 & A_{15} & 1 & 0 & 1 & 0 & 1 & A_{25} & 0 & 1 & 1 & 1 & 1 \\ A_{6} & 1 & 1 & 1 & 1 & 0 & A_{16} & 1 & 0 & 0 & 0 & 0 & A_{26} & 0 & 1 & 1 & 0 & 0 \\ A_{7} & 1 & 0 & 0 & 0 & 1 & A_{17} & 1 & 0 & 1 & 0 & 0 & A_{27} & 1 & 0 & 1 & 1 & 1 \\ A_{8} & 1 & 1 & 1 & 1 & 1 & A_{18} & 1 & 1 & 0 & 0 & 1 & A_{28} & 0 & 0 & 0 & 0 \\ A_{9} & 0 & 1 & 0 & 1 & 0 & A_{19} & 0 & 0 & 1 & 1 & 1 & A_{29} & 1 & 0 & 0 & 1 & 0 \\ & & & & & & & & & & A_{30} & 0 & 0 & 1 & 1 & 0\end{array}$

Fig. 3. "Kendall sequence" for the polynomial $x^{5}+x^{2}+1$. 


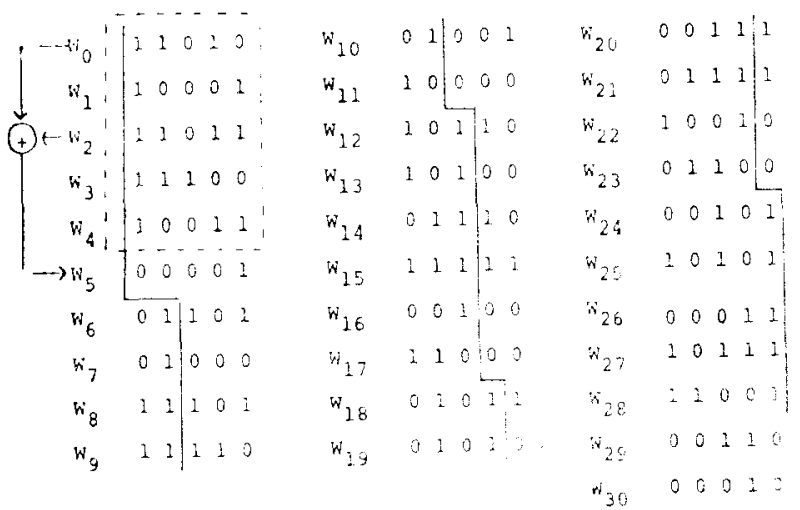

Fig. 4. GFSR sequence for polynomial $x^{5}+x^{2}+1$ with delay $-6(=25)$ between each column.

$\left(W_{0}, W_{1}, W_{2}, W_{3}, W_{4}\right.$ for $\left.x^{5}+x^{2}+1\right)$ can be checked to see if this desirable property holds, and second, the number of different sequences with this property can be analytically computed.

For clarity, we will proceed by using the example in Fig. 4. The companion matrix for polynomial $x^{5}+x^{2}+1$ is

$$
\subseteq=\left[\begin{array}{lllll}
0 & 0 & 0 & 0 & 1 \\
1 & 0 & 0 & 0 & 0 \\
0 & 1 & 0 & 0 & 1 \\
0 & 0 & 1 & 0 & 0 \\
0 & 0 & 0 & 1 & 0
\end{array}\right]
$$

so

$\begin{array}{lllll}w_{0} & w_{1} & w_{2} & w_{3} & w_{4}\end{array}$

$\left[\begin{array}{lllll}1 & 1 & 1 & 1 & 1 \\ 1 & 0 & 1 & 1 & 0 \\ 0 & 0 & 0 & 1 & 0 \\ 1 & 0 & 1 & 0 & 1 \\ 0 & 1 & 1 & 0 & 1\end{array}\right]\left[\begin{array}{lllll}0 & 0 & 0 & 0 & 1 \\ 1 & 0 & 0 & 0 & 0 \\ 0 & 1 & 0 & 0 & 1 \\ 0 & 0 & 1 & 0 & 0 \\ 0 & 0 & 0 & 1 & 0\end{array}\right]=\left[\begin{array}{lllll}1 & 1 & 1 & 1 & 0 \\ 0 & 1 & 1 & 0 & 0 \\ 0 & 4 & 1 & 0 & 0 \\ 0 & 1 & 0 & 1 & 0 \\ 1 & 1 & 0 & 1 & 1\end{array}\right]$

or in matrix notation $\mathrm{W}_{0} \mathrm{C}=\mathrm{W}_{1}$. After $2^{5}-1=31$ applications of this matrix recurrence, $\mathrm{W}_{0} \mathrm{C}^{31}=\mathrm{W}_{0}$ or $\mathrm{C}^{31}$ is congruent modulo 2 to the identity matrix. Clearly, $\mathrm{C}, \mathrm{C}^{2}, \mathrm{C}^{3}, \cdots$, and $\mathrm{C}^{30}$ are all different by virtue of the period of the shift register sequence. Thus, for all $\mathrm{W}_{0} \mathrm{C}^{\mathrm{k}}, 1<\mathrm{k}<30$, to be different, it is only necessary that $W_{0}$ have linearly independent rows. This is easily checked.

The number of different sequences which can be produced by the GFSR algorithm is equal to the number of ways different $W_{0}$ matrices with linearly independent rows can be selected.

Let $r_{1}, r_{2}, r_{3}, r_{4}$, and $r_{5}$ denote the five rows of Matrix $W_{0}$. Row $r_{1}$ can be selected to be any of the $2^{5}-1=31$ nonzero binary vectors. Row $r_{2}$ can be any of the $2^{5}-1-1=30$ binary vectors which excludes $r_{1}$ and the zero vector. Row $r_{3}$ can be any of the $2^{5}-1-3=28$ binary vectors which excludes the zero vector and any linear combination of $I_{1}$ and $r_{2}$. Row $r_{4}$ can be any of the $2^{5}-1-7=24$ binary vectors excluding the zero vector and linear combinations of $r_{1}$, $r_{2}, r_{3}$. The same argument can be extended to selection of $r_{4}$. Thus, with the GFSR algorithm, it is possible to produce $\left(2^{5}-1\right)\left(2^{5}-2\right)\left(2^{5}-4\right)\left(2^{5}-8\right)\left(2^{5}-16\right)=$ $(31)(30)(28)(24)(16)=9,999,360$ different sequences based on primitive polynomial $x^{5}+x^{2}+1$.

The GFSR algorithm is,

GFSR: 0 . if $k \neq 0$, go to 2 ( $k$ initially zero).

initialize, $\mathrm{W}_{0}, \cdots, \mathrm{W}_{\mathrm{p}-1}$ using a delayed basic sequence $\left(a_{i}\right)$ to obtain each column of $\mathrm{W}_{0}, \cdots, \mathrm{W}_{\mathrm{p}-1}$.

2. $k \leftarrow k+1$

3. if $k>$ p. set $k \leftarrow 1$

4. $\mathrm{j} \leftarrow \mathrm{k}+\mathrm{q}$.

5. if $\mathrm{j}>\mathrm{p}$, set $\mathrm{j} \leftarrow \mathrm{j}-\mathrm{p}$.

6. exclusive-or, $W_{k} \oplus W_{j}$.

7. store, $W_{k} \leftarrow W_{k} \oplus W_{j}$.

A rotating table of $\mathrm{p}$ words is kept in GFSR, which is implemented as a FORTRAN function in Fig. 5. A FORTRAN function allows complete generality to be realized, as shown by the results for IBM 360/67, SRU 1108, CDC 6400, and HP 2116A seen in Fig. 6. Although the compiled FORTRAN code of Fig. 5 correctly implemented RAND for these four computers, some compilers may not implement full word logical operations, or others may optimize logical computations. Nevertheless, GFSR pseudorandom numbers may be checked for validity against the results in Fig. 6.

\section{INITIALIZATION OF GFSR ALGORITHM}

The GFSR algorithm is self-initializing in the sense that delayed replicas are produced by the same procedure that generates full words. Linear independence of starting columns is guaranteed if the maximum delay measured from the leftmost column is less than the full period, $2^{\mathrm{p}} \ldots 1$ (if the "constant delay"

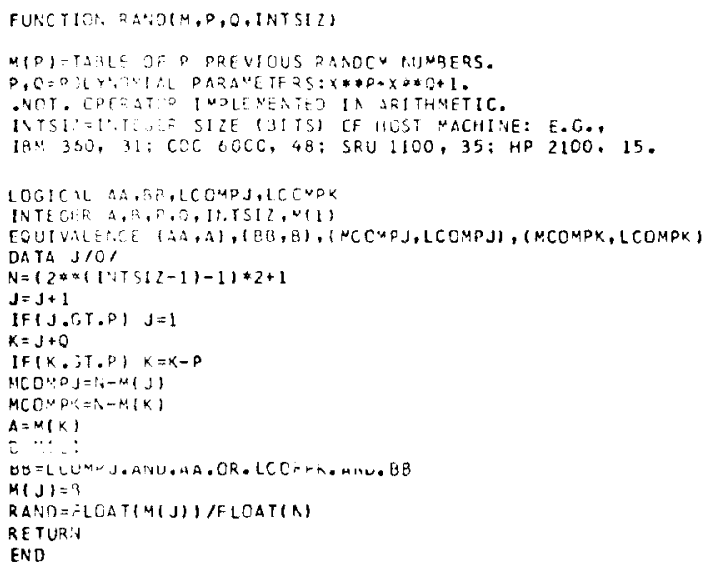

Fig. 5. FORTRAN implementation of GFSR algorithm. Initialization is done by SETR in Fig. 7. 


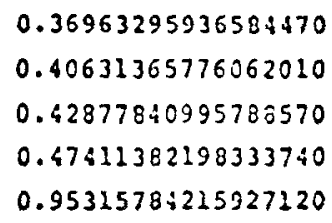

(a)

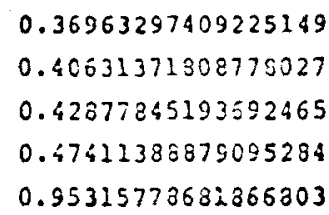

(c)
0.369632978 .00000000

0.40631372300000000

0.42877845000000000

0.47411389000000000

0.95315778000000000

(b)

0.36964017152786255

0.40632343292236328

0.47420506320055542

(d)
0.42878508567810059

0.95318460464477539

Fig. 6. The first five normalized pseudorandom numbers from GFSR: $x^{98}+x^{27}+1$, delayed column $=9800$, produced on the (a) IBM 360, (b) SRU 1108, (c) CDC 6400, and (d) HP 2116 computers.

between each column is relatively prime to $2^{p}-1$, then maximum delay can exceed the full period). Using this procedure, every p-tuple is generated (except all zeros) before any p-tuple repeats. Each initial column is a p-tuple, and therefore must be independent of all others. For example, in Fig. 4, initialization can be done from most significant to least significant bits (left-to-right), starting with [11111]. The recurrence relation is applied 25 times, here, to get the next column [10110]. A second 25 applications results in [00010], a third 25 applications results in [1010i] ; and rinaily, [0 010101$]$ is obtained. The recurrence is applied by calling the GFSR RNG with zero fills placed to the right. Each new column is shifted to the right after being generated, and the original column [11111] is replaced at the extreme

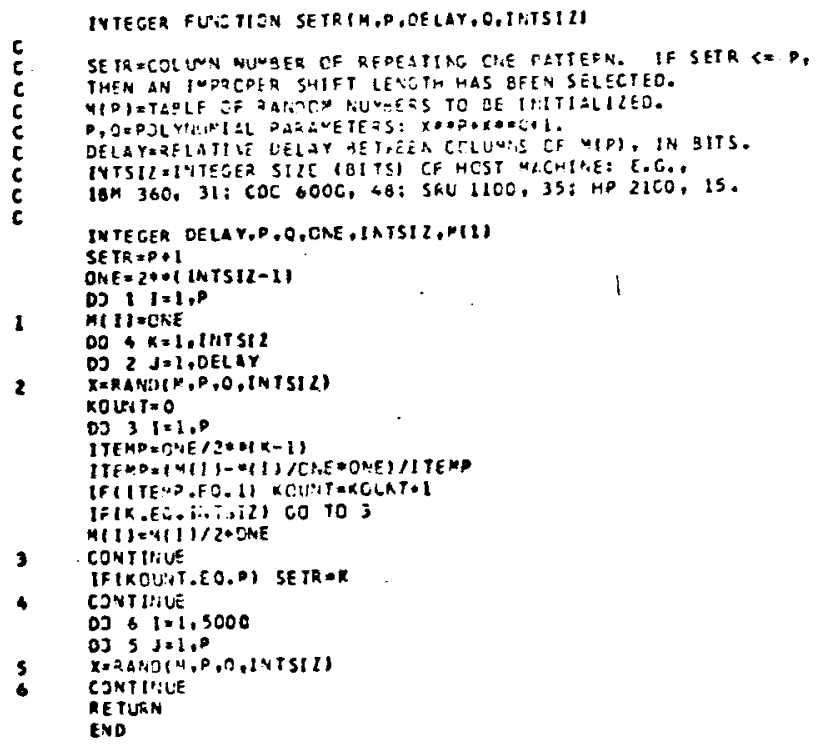

Fig. 7. FORTRAN implementation of SETR to initialize GFSR algorithm. left column. In more realistic (bigger word size) generators. the full period will not be exhausted by initialization in keeping with conditions for linear independence (here. linear independence was guaranteed, because 25 is relatively prime to 31 ).

For ease in implementation. the [111..] starting p-tuple is used in SETR, as given in Fig. 7. However, SETR applies 5000p additional delays so that the leading $[111 \ldots]$ column is "recurrenced" also, thus giving a randon pattern of leading bits rather than all ones. This additional "recurrencing" is necessary to allow $[111 \ldots]$ to "die out." The effects of such a regular pattern carry over to later p-tuples. For example, $[111 \ldots], p=98, q=27$, is transformed to 72 zeros, 26 ones; next to 45 zeros, 53 ones, etc. The groupings of all ones or all zeros becomes increasingly smaller and more "random" through repeated application of the recurrence relation. "Damping" of the initial p-tuple is done in SETR by applying the recurrence relation 5000p

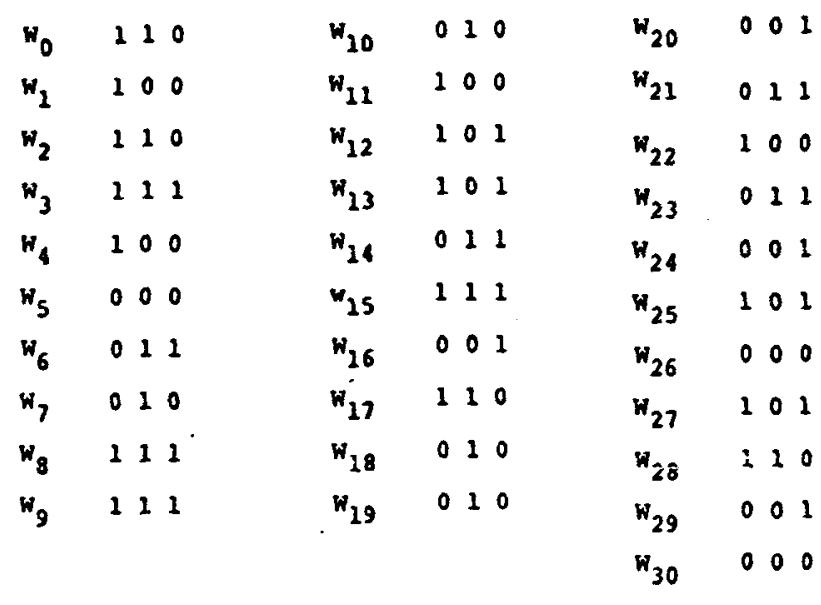
1.

Fig. 8. GFSR algorithms for $L=3$ with polynomial $x^{5}+x^{2}+$

times to full-word starting values. It should be noted that if generality relative to word size is not desired, then additional "recurrencing" is not necessary when initialization is performed from least significant to most significant bits. The most significant bit will have been delayed p DELAY times, since it is "recurrenced" once for each bit in a p-bit word (the least significant bit is a one). Thus, the additional "randomizing" of the initial $[111 \ldots]$ pattern will have been done without additional labor.

Finally, SETR returns a value for the number of linearly independent columns available to the initialization procedure.

\section{Generality of GFSR}

The parallel nature of GFSR immediately generalizes to L-bit (integer size) machines independent of the relation between $\mathrm{L}$ and $\mathrm{p}$. Thus, for $\mathrm{L}<\mathrm{p}$, many repeated numbers will occur, but cycle length, $m$, is still $2^{p}-1$. The case where $L=3$ and $x^{5}+x^{2}+1$, from 


$\begin{array}{ll}p & q \\ 47 & 5,14,20,21 \\ 95 & 21,27 \\ 98 & 11,27 \\ 211 & 10,45 \\ 224 & 37 \\ 170 & 23 \\ 250 & 203 \\ 380 & 47 \\ 476 & 25,141 \\ 532 & 37\end{array}$

Fig. 9. Primitive polynomials, $x^{p}+x^{q}+1, p$ large.

Fig. 4, is demonstrated in Fig. 8. Here, $2^{\mathrm{p}-\mathrm{L}}$ nonzero duplicates and $2^{\mathrm{p}-\mathrm{L}}-1$ zeros are produced in one full period.

Very long period sequences can be generated on any L-bit machine merely by selecting p large. A partial table of primitive polynomials of large $p$ is reproduced in Fig. 9 (Whittlesey \& Griese, 1970). A complete table can be found in Zierler (1969) and Zierler and Brillhart (1969).

\section{Period of GFSR}

An "unlimited" period is possible without increasing word size of host machines. For example, $M=2^{532}-1$ is obtained using $x^{532}+x^{37}+1$ from the table in Fig. 9. To exhaust this cycle would require many years on a very fast computer, i.e., if $10^{6}$ numbers $/ \mathrm{sec}$ were generated. approximately $10^{150}$ years would be needed to complete the cycle! More importantly, though. is the repeatability of numbers within a full period. Thus, an extended sequence is obtained with desirable n-space properties.

\section{Multidimensional Uniformity of GFSR}

Figure 10 shows a much improved 9-bit generator compared with Lehmer and Kendall RNGs shown in Figs. 1 and 2. The underlying reason, of course, is the longer than $m=2^{9}$ period, and the repeatability of numbers within one cycle of the generator.
I. i.i. $m^{\text {r }}$ cells in $n$ space, $\left(2^{L}\right)^{n}<2^{p}-1$. or 11. - Therefore. a necessary condition for $n$-space unitiormity is that $n<p / L$. For example, suppose $L=15$ and $p=98$. then uniformity may be possible up to dimension $n=6$.

\section{GFSR Theorem}

The sequence of L-bit numbers generated by GFSR, $\mathrm{x}^{\mathrm{p}}+\mathrm{x}^{\mathrm{q}}+1, \mathrm{p}>\mathrm{L}$, has: (1) period, $\mathrm{m}=2^{\mathrm{p}}-1$, if $\mathrm{x}^{\mathrm{p}}+$ $\mathrm{x}^{\mathrm{q}}+1$ is primitive, $(2)$ normalized mean, $\mu_{0} \approx 1 / 2$, (3) normalized variance, $\sigma_{0}^{2} \approx 1 / 12$, (4) potential n-space uniformity for $\mathrm{n}<\mathrm{p} / \mathrm{L}$.

\section{Testing}

In addition to analytic calculations of the mean, variance, and correlations for the full period sequence (Lewis \& Payne, 1973), the following empirical tests on 10,000 GFSR produced numbers were made using $x^{98}+$ $\mathrm{X}^{27}+1,15$ bits, and chi-square statistic (Kendall \& Smith, 1938: Lewis \& Payne, 1973; Marsaglia, 1968).

Frequency test counts the number of numbers falling in each of 100 equal cells in the $(0,1)$ interval.

Yule's test counts the number of sums-of-5 digits falling in each of 45 cells. This is applied to each of the four most significant decimal digits obtained by scaling the normalized $(0,1)$ pseudorandom number.

Gap test counts the length of gaps between successive-like digits formed by scaling normalized RNG number to decimal digits. The test is applied to digits 0 through 9 .

Autocorrelation test computes the maximum normalized autocorrelation coefficient up to lag 50 . Acceptable correlation is in the interval $(.03, .08)$.

$D^{2}$ test compares the theoretical distribution of a random line in two dimensions with the distribution obtained empirically.

Serial test counts the number of pairs of numbers from a RNG falling in 100 equal cells. Here, pairs of decimal digits are combined to give an integer from 0 to 99. A frequency test is applied to the 100 cells.

Runs test computes the longest run, the frequency of each run compared with the theoretical distribution, and number of runs above/below the mean. Total number of runs is compared with the expected number.
Fig. 10. (a) Two-dimensional plot of GFSR. $X^{31}+X^{13}+1,9$-bit word size and delay of 93: (b) three-dimensional plot of GFSR, $X^{31}+X^{13}+1$. delay $=93$.

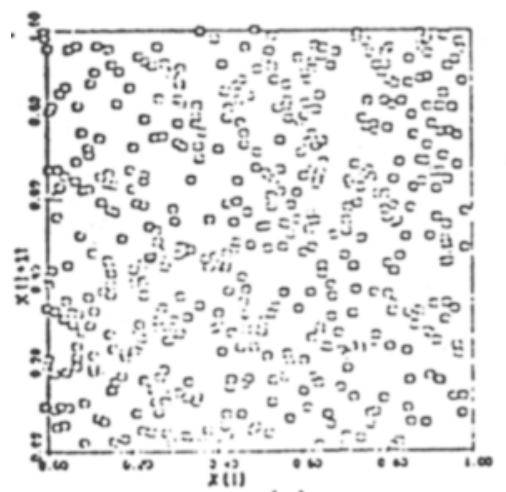

(a)

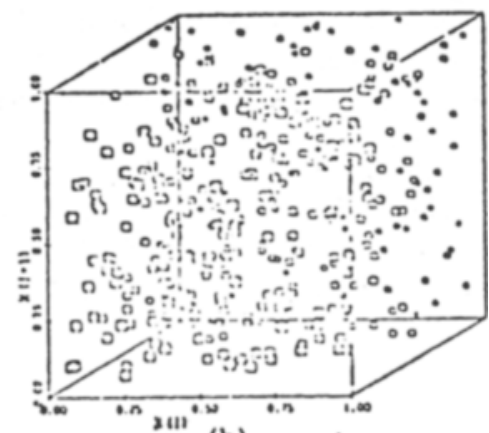

(b) 
The minimum/maximum-of-n test compares the empirical with the theoretical distribution of the minimum/maximum-of $n$ numbers. The number of extremes are counted for each of 100 cells and a frequency test applied for $n=2,4.6 . \cdots, 20$.

The conditional bit test counts the number of one bits in the $\mathrm{j}^{\text {th }}$ bit position given the $\mathrm{j}-1$ previous bits. $\mathrm{A}$ binary tree is formed with a branch at each bit position. The tree of bit counts is then compared with the expected value of $(1 / 2) * 10.000=5.000$. If the bits are indeed independent, then the empirical value matches expected value.

The finite Fourier transform (FFT) test tests for a "flat" spectrum by the statistics:

$$
\begin{aligned}
U= & {[S-(1 / 2)] \sqrt{12 M} } \\
S= & (1 / M) \sum_{n=0}^{M} P_{n} \\
P_{n}= & \sum_{r=1}^{n}\left|a_{n}\right|^{2} / \sum_{r=1}^{M H}\left|a_{n}\right|^{2}, n=1,2, \cdots M+1 \\
M= & N / 2 ; N \text { even } \\
& (N-3) / 2 ; N \text { odd }
\end{aligned}
$$

and the transformed sequence:

$$
\begin{aligned}
& A_{n}=(1 / N) \sum_{j=0}^{N-1} R_{j} \exp (-2 \pi i j n / N) ; n=0,1, \cdots N-1 \\
& R_{j}=\text { random number }
\end{aligned}
$$

The scattering experiment simulation compares the expected distribution of scattering angles with theoretically expected values. A point on the surface of a unit sphere is randomly chosen, thus giving a solid angle. This solid angle represents the deflection of a neutron after colliding with an atom. The solid angle is rejected to weight according to $\sigma(\Theta)=\pi(1-\Theta / \pi): 0 \leqslant$ $\Theta \leqslant \pi$. The empirical distribution of $\Theta$ is compared with the theoretical, using chi-square test on 36 intervals in $(0, \pi)$.

A visual test is done by observing 2,3-space plots of successive n-tuples, as shown in Fig. 10. A CRT display is particularly useful for observing order by dynamic plotting of points as they are generated.

\section{Results of Tests}

The 15-bit GFSR algorithm performed satisfactorily in the above tests at the $5 \%$ level of significance. Failure was noted at $n=8,14,20$ in the minimum-of-n test, as predicted. However, no failures were noted for $n=10$. 12. 16. 18, and all $\mathrm{n}$ tested in the maximum-of- $\mathrm{n}$ test. Thus a partial indication of nonuniformity for $n>6$ is given by these tests. For applications requiring uniformity above $n=6$. one merely uses a higher degree polynomial. For 24 mantissa bits and $X^{98}+X^{27}+1$, no failure was detected on any of the tests.

The GFSR algorithm must be considered statistically qualified as a RNG, while superior in speed and generality to other contemporary RNGs.

\section{CONCLUSIONS}

The advantages of GFSR RNG are: (1) Speed. One exclusive-or vs a multiply/reduction modulo $\mathrm{m}$ for Lehmer, and two exclusive-ors/two shifts for Kendall's algorithm. (2) Generality. A standard FORTRAN subprogram can be implemented on any computer independent of the word size. A small word size merely reduces the resolution of random numbers produced, but high-order bits will be unchanged on any machine. Comparison with sequences obtained on other machines using a FORTRAN program are given in Fig. 6. (3) "Unlimited" period. Any primitive polynomial can be implemented when sufficient memory is available for storage of $\mathrm{p}$ words. For example, $\mathrm{x}^{98}+\mathrm{x}^{27}+1$ can be used on a 16-, 24-, 32-, 36-, 48-, 60-bit machine and a cycle length of $2^{98}-1$ realized on them all.

\section{REFERENCES}

unifor uniform random number generators. Journal of the Association for Computing Machinery, 1967, 14, 100-119.

Kendall, M. G., \& Smith, B. B. Randomness and random sampling numbers. Journal of the Royal Statistical Society, $1938,101,162-164$.

Lewis, T. G., \& Payne, W. H. Generalized feedback shift register pseudorandom number generator. Journal of the Association for Computing Machinery, 1973, in press.

MacLaren, $M$. V., \& Marsaglia, G. Uniform random number generators. Journal of the Association for Computing Machinery, $1965,12,83-89$.

Marsaglia, G. Random numbers fall mainly on the planes. Proceedings of the National Academy of Sciences, 1968,61, 25-28

Payne, W. H., \& Lewis, T. G. Conditional bit sampling: Accuracy and speed. In J. R. Rice (Ed.), Mathematical software. New York: Academic Press, 1971.

Tausworthe, R. C. Random numbers generated by linear recurrence modulo two. Mathematical Computations, 1965, 19, 201-209.

Tootill, J. P. R., Robinson, W. D., \& Adams, A. G. The runs up-and-down performance of Tausworthe pseudorandom number generators. Journal of the Association for Computing Machinery, 1971, 18, 381-399.

Whittlesey, J. R. B. A comparison of the correlation behavior of random number generators for the IBM 360. Communications of the Association for Computing Machinery, 1968, 11, 641-644.

Whittlesey, J. R. B., \& Griese, P. Multi-dimensional pseudorand om non-uniformity. Proceedings of the UMR-Mervin J. Kelly Communication Conference. October $1970,15-y-1-15 \cdot 4-6$

Zierler, N. Primitive trinomials whose degree is a Mersenne exponent. Information \& Control, 1969, 15, 67-69.

Zierler, N., \& Brillhart, J. On primitive trinomials (mod 2), II. Information \& Control, $1969,14,566-569$. 